Check for updates

Cite this: RSC Adv., 2017, 7, 41593

Received 28th May 2017

Accepted 3rd August 2017

DOI: $10.1039 / c 7 r a 05966 c$

rsc.li/rsc-advances

\section{Preparation and biocompatibility of electrospinning PDLLA/ $\beta$-TCP/collagen for peripheral nerve regeneration}

\author{
Fei Lin, ${ }^{\text {ab }}$ Xinyu Wang, (D) *ab Yiyu Wang, ${ }^{\text {ab }}$ Yushi Yang ${ }^{\text {ab }}$ and Yi Lic
}

A unique nerve conduit composed of poly(D,L-lactic acid) (PDLLA), $\beta$-tricalcium phosphate ( $\beta$-TCP) and collagen was prepared by electrospinning for the first time. Scanning electron microscopy (SEM) showed that the mean diameters of the pores in the nerve conduits were less than $10 \mu \mathrm{m}$ and greater than $1 \mu \mathrm{m}$. These conduits had bionic structures, larger porosity and relatively higher surface area than traditional nerve conduits, thus not only permitting the transportation of nerve growth factor and glucose but also blocking the entry of lymphatic tissue and fibroblasts. The uniform fibers of nerve conduits can mimic the natural extracellular matrix, which is beneficial to cell adhesion, cell proliferation and cell migration. PDLLA/ $\beta$-TCP/collagen overcomes the challenge of low degradation rate, and maintains the basic $\mathrm{pH}$ environment for cell growth. PDLLA/ $\beta$-TCP/collagen showed good cellular affinity and a low hemolytic rate, which is beneficial for RSC96 Schwann cells adhesion, migration and proliferation. Its good biocompatibility enables its safety in in vivo tests. A $10 \mathrm{~mm}$ sciatic nerve defect was bridged in vivo with PDLLA/ $\beta$-TCP/collagen conduits (c) in Wistar rats. PDLLA groups (a) and PDLLA/ $\beta$-TCP groups (b) were set and autotransplants (d) were used as control groups. Compared to a and b, c achieved significantly more effective regeneration of sciatic nerve injuries after 3 months' implantation and the mean diameter of soleus muscle fibers in c $(39.62 \pm 3.91 \mu \mathrm{m})$ was 1.28 times larger than that in $\mathrm{b}(30.89 \pm 5.89 \mu \mathrm{m})$, and it was closer to that in $\mathrm{d}$. The regenerated nerve fibers in $\mathrm{c}$ had a more regular round shape, the density of neurofilaments in $c$ was more than those in $a$ and $b$, and was close to that in $d$. PDLLA/ $\beta$-TCP/ collagen has a bionic structure, good cellular affinity and biodegradability, which can overcome the drawbacks of the current nerve conduits; hence, it holds great promise for effective of sciatic nerve regeneration.

\section{Introduction}

In recent years, with the increase in cases of nerve injuries, nerve grafts couldn't meet the demands of neural restoration. Autografts in peripheral nerve injuries have inevitable drawbacks in the repair of injured nerves, particularly in the vast area of injuries to peripheral nerves. ${ }^{1}$ Autografts are often ineffective because of the gap length between the injured nerves, formation of neuromas, and shortage of donor sources. ${ }^{2}$ Complete restoration of function in damaged nerves will significantly improve the lives of affected individuals and reduce the associated socioeconomic costs. ${ }^{3}$ To achieve such restoration, various types of artificial nerve conduits that connect the ends

${ }^{a}$ State Key Laboratory of Advanced Technology for Materials Synthesis and Processing, Wuhan University of Technology, Wuhan 430070, P. R. China. E-mail: wangxinyu@ whut.edu.cn; Fax: +86-27-87880734; Tel: +86-27-87651853

${ }^{b}$ Biomedical Materials and Engineering Research Centre of Hubei Province, Wuhan 430079, P. R. China

'Institute of Textiles and Clothing, The Hong Kong Polytechnic University, Hong Kong, 999077, P. R. China of injured peripheral nerves were developed in order to provide micro-environment and growth orientation for the nerve cells. ${ }^{4}$ Nerve conduits have sufficient material source and can repair even a long-distance nerve injury; however, the mechanical properties and the biological activity of nerve conduits are poor. Therefore, preparing excellent nerve conduits is still challenging. ${ }^{5,6}$

To avoid unnecessary surgery, the nerve conduits to be implanted in vivo should be biocompatible and biodegradable. ${ }^{7}$ As a result, more attention has been placed on the research of synthetic nerve conduits with different biomaterials; the material of these conduits should also be biocompatible and biodegradable. Currently, materials such as PLLA, ${ }^{8}$ genipincross-linked gelatin, ${ }^{\mathbf{9}}$ collagen, ${ }^{\mathbf{1 0}, \mathbf{1 1}}$ silicone, ${ }^{\mathbf{1 2}}$ gelatin, ${ }^{\mathbf{1 3}}$ laminin, ${ }^{\mathbf{1 4}}$ and chitin have been used to prepare nerve conduits. PDLLA is widely applied in drug delivery and tissue engineering scaffold, ${ }^{15,16}$ due to its good biocompatibility and mechanical properties; however, its poor cellular affinity limits application in tissue engineering. Dawei Li et al. ${ }^{17}$ reported the technique of electrospinning P(LLA-CL) nanofibers and PLLA nanofiber yarns, which is used for nerve repair, but it showed poor cell 
compatibility; hence, the addition of collagen is very important for improving the cell compatibility of nerve conduits. Tong $\mathrm{Wu}$ et al. ${ }^{18}$ prepared a conduit that contains laminin (LC-YE-PLGA NGC) to promote SC's proliferation and migration. Collagen has an abundant amount of laminin, which has good cellular affinity, which in turn can mediate cell adhesion, migration and proliferation. Low antigenicity and cytotoxicity of collagen play an important role in vivo and no significant rejection reactions between tissues and nerve conduits; however, the mechanical properties of collagen are poor, ${ }^{19}$ because of which it is unable to sustain its morphology and size in nerve conduits. The ideal nerve conduit should also have appropriate mechanical properties in order to provide a suitable stress environment. In addition, such a conduit should be porous and permeable in order to permit the ingress of cells and nutrients. ${ }^{20}$

Several methods such as freeze-drying, ${ }^{21,22}$ sol-gel, ${ }^{23}$ biopolymer replication ${ }^{24-26}$ and gas foaming ${ }^{27}$ have been utilized to fabricate nerve conduits. Among these methods, electrospinning appears to be a simple and effective choice to produce conduits composed of polymer fibers. Such conduits have large porosity and surface area; hence, they are suitable as scaffolds for tissue engineering..$^{28}$ Busra Mammadov et al. ${ }^{29}$ successfully used the electrospinning method for preparing glycosaminoglycan and laminin mimetic peptide nanofiber gels, which present a promising treatment alternative for peripheral nerve injuries. Esmaeil Biazar et al. ${ }^{30}$ electrospun a nanofibrous PHBV conduit to bridge a $30 \mathrm{~mm}$ sciatic nerve defect in a rat model of sciatic nerve injury, which was repaired successfully. Electrospinning combined with the rotor drum receiving method was used to fabricate nerve conduits (NCs) with PLGA-silk fibroincollagen (PLGA-SF-CL) and PLGA, which successfully bridged a $10 \mathrm{~mm}$ sciatic nerve defect. ${ }^{31}$ In particular, these fibrous structures can mimic the natural extracellular matrix of nerve cells. As a result, the adhesion, proliferation and migration of these cells are improved, which is helpful for the repair of peripheral nerves. ${ }^{32}$

In this study, poly(D,L-lactic acid) (PDLLA) was chosen as the host material on account of its novel biodegradability, biocompatibility and mechanical properties. However, the degradation of PDLLA is accompanied by a decrease in the $\mathrm{pH}$ of the ambient environment, which is unfavorable for the cells. ${ }^{33}$ In order to fix this issue, beta tri-calcium phosphate ( $\beta$ TCP) was used to neutralize the acidic environment. ${ }^{34,35}$ Moreover, the cellular affinity of PDLLA was poor and PDLLA scaffolds could not connect with the polypeptide (RGD) due to the lack of cell identification signals. ${ }^{36-39}$ To increase such affinity, collagen was used to modify PDLLA due to the fact that collagen is capable of enhancing the adhesion and migration of nerve cells $^{40-42}$ as well as guiding axon elongation. ${ }^{43-46}$ As an extra benefit, collagen can also enhance the flexibility of the scaffold $^{47,48}$ at the cost of lowering the overall mechanical strength. ${ }^{49,50}$

The academic significance of the current study is very important in that the composite conduits of PDLLA/ $\beta$-TCP/ collagen have been prepared via electrospinning for the first time. These conduits can overcome the drawbacks of acid degradation and weak cell affinity of traditional nerve conduits.
The addition of $\beta$-TCP and collagen can neutralize the acid generated by PDLLA, which is favorable to nerve regeneration. The electrosurgical conduit of PDLLA/ $\beta$-TCP/collagen has changed the single structure of the traditional nerve conduits, which have a bionic structure that can permit the transportation of nerve growth factor and glucose, but blocks the entry of lymphatic tissue and fibroblasts.

Composite materials with the advantages of biodegradability and biocompatibility are used to imitate the structure and function of natural nerves ${ }^{9,13,50-53}$ and are commonly used for several biomedical applications..$^{54}$ Combined with the advantages of PDLLA, $\beta$-TCP and collagen, composite nerve conduits for peripheral nerve regeneration were prepared through electrospinning, and they had excellent mechanical properties, biocompatibility, and neutral microenvironment. PDLLA can enhance the mechanical properties and improve cell affinity. PDLLA and collagen produce an acidic substance during degradation, which causes a $\mathrm{pH}$ decline that is harmful to cell growth. However, $\beta$-TCP can upregulate mRNA expression of cytoskeletal protein and neutralize the acidic substance; hence, the $\beta$-TCP composite with PDLLA and collagen can adjust the $\mathrm{pH}$ of the microenvironment in nerve conduits. ${ }^{55}$

In this article, PDLLA was purchased and polymer films composed of pure PDLLA, PDLLA $/ \beta$-TCP and PDLLA $/ \beta-T C P /$ collagen were prepared via electrospinning. These films were rolled up and stuck to make the nerve conduits. The conduits were analyzed in terms of the material property, mechanical property and biodegradation using scanning electron microscopy (SEM). We used MTT and the hemolytic test method to assess the material toxicity and hemolytic rate. In order to study the feasibility of this composite conduit, a $10 \mathrm{~mm}$ sciatic nerve defect was bridged with PDLLA/ $\beta$-TCP/collagen conduits in Wistar rats. PDLLA and PDLLA/ $\beta$-TCP were set and autotransplants were used as control groups. The regenerated nerves were analyzed by hematoxylin-eosin and immunofluorescence staining. The study plan was approved by the Animal Care and Use Committees of Wuhan University of Technology, China and performed in accordance with the National Institutes of Health Guide for the Care and Use of Laboratory Animals (no. 85-23, revised 1996). In this article, we analyzed the feasibility of this composite conduit from material property, cell and animal experiments.

\section{Experimental section}

\section{Materials}

PDLLA, $\beta$-TCP, dichloromethane, ethyl acetate and dimethyl sulfoxide (DMSO) were purchased from Sinopharm Chemical Reagent Co. Ltd (China). Phosphate buffered saline (PBS), collagen and MTT were purchased from Soho Biotechnology Co. Ltd. (China). Anti-neurofilament 200, hematoxylin and eosin and DAPI were purchased from Beyotime Institute of Biotechnology (China). Collagen (type I) was purchased from Biosharp (from Bovine Achilles Tendon). All other reagents were of analytical grade. 


\section{Fabrication of the nerve conduits}

The electrospinning solution of pure PDLLA was prepared by dissolving PDLLA $(\mathrm{w} / \mathrm{v} \%=10 \%)$ in a mixture of dichloromethane and ethyl acetate (volume ratio $=7: 3$ ). In order to obtain the PDLLA/ $\beta$-TCP film, excess of $\beta$-TCP $(\mathrm{w} / \mathrm{v} \%=3 \%)$ was added into the PDLLA solution. For the PDLLA $/ \beta-T C P /$ collagen film, collagen $(\mathrm{w} / \mathrm{v} \%=3 \%)$ was added to the PDLLA $/ \beta$-TCP solution. The electrospinning solution was loaded into a syringe with an end nozzle controlled by a syringe pump at a feeding rate of $0.8 \mathrm{~mL} \mathrm{~h}^{-1}$. A DC voltage of $+3 \mathrm{kV}$ was applied to the nozzle. The ejected fibers were collected on a collector covered with aluminum foil. A voltage of $-3 \mathrm{kV}$ was applied to the collector. The distance between the nozzle and the collector was $15 \mathrm{~cm}$. After electrospinning, the as-obtained polymer films were placed in a vacuum drying oven for $72 \mathrm{~h}$ at $40{ }^{\circ} \mathrm{C}$ to remove residual solvent. The films of pure PDLLA, PDLLA/ $\beta$-TCP and PDLLA/ $\beta$-TCP/collagen were labeled as sample $a, b$ and $c$, respectively. These films were pasted into conduits with dichloromethane. All these conduits were sterilized with ethylene oxide and further irradiated with ultraviolet light before further assessments to overcome the environmental and other factors that could affect the experimental results. The experiments were stringent in terms of scientific operation to maintain safety during the process of the experiment. We also set a large number of control groups so as to ensure the objectivity of the experiment.

\section{Material characterization}

The composition of sample a, b and c were characterized by Fourier transform infrared spectroscopy (FTIR) using an FTIR spectrometer (Nexus, USA). The morphology of the polymer films was observed with scanning electron microscopy (SEM, Hitachi S-4800, Japan). The mechanical strength of the samples was characterized by a universal material testing machine (Instron 5848, USA). To monitor the degradation of the polymer films, samples were incubated in phosphate buffer solution (pH: $7.4 \pm 0.2$ ) at $37^{\circ} \mathrm{C}$. The mass loss of the samples and the $\mathrm{pH}$ value of the PBS solution were recorded on a weekly basis.

\section{MTT}

This test was conducted according to the standard of $\mathrm{GB} / \mathrm{T}$ 168865 -2003. The leaching solution of materials is $0.2 \mathrm{~g} \mathrm{~mL}^{-1}$. The films of (a) PDLLA, (b) PDLLA/ $\beta$-TCP and (c) PDLLA $/ \beta$-TCP/ collagen were films of (a) PDLLA, (b) PDLLA/ $\beta$-TCP and (c) PDLLA $/ \beta$-TCP/collagen were added to a liquid mixture (DMEM (GIBCO)) in different centrifuge tubes with 10\% FBS (GIBCO), $1.0 \times 10^{5} \mathrm{mg} \mathrm{L}^{-1}$ penicillin (Sigma), and $100 \mathrm{mg} \mathrm{L}^{-1}$ streptomycin (Sigma), respectively, which was extracted then after $24 \mathrm{~h}$ at $37{ }^{\circ} \mathrm{C}$, which afforded the leaching solution of the films. RSC96 cells were seeded on 96-well plates at a density of 2000 cells per $\mathrm{cm}^{2}$; the upper culture was removed after the RSC96 cells adhered on the bottom. Then, $100 \mu \mathrm{L}$ leaching solution of different films were added into each 96-well plate and set six parallel samples. In addition, positive groups (DMSO) and negative groups (blank control), as control groups, were put into 96-well plates in a humidified incubator at $37{ }^{\circ} \mathrm{C}$ and $5 \% \mathrm{CO}_{2}$ after adding the leaching solution. After an incubation time of $1 \mathrm{~d}, 3 \mathrm{~d}$ and $5 \mathrm{~d}$, the supernatant of the wells was removed. MTT solution $\left(20 \mu \mathrm{L} ; 5 \mathrm{mg} \mathrm{mL}^{-1}\right.$ in PBS) was added and incubated for $4 \mathrm{~h}$ at a temperature of $37{ }^{\circ} \mathrm{C}$ and $5 \% \mathrm{CO}_{2}$. Then $100 \mu \mathrm{L}$ DMSO was added into each well with a $10 \mathrm{~min}$ oscillation of the shaker. The optical absorbance at $490 \mathrm{~nm}$ was recorded by the automatic enzyme instrument (Thermo Labsystems 1500-206, USA), and the average optical density (OD) values of six parallel samples as experimental values were calculated.

\section{Hemolytic test}

The samples of the three groups were cut into small pieces and placed in three centrifuge tubes. $10 \mathrm{~mL}$ PBS was added into each centrifuge tube and the tubes were placed in a thermostatic water bath at $37^{\circ} \mathrm{C}$ for $24 \mathrm{~h}$, after which the leaching solution was obtained. Fresh blood was collected from the vein of rabbit ear, putting the blood into anticoagulant which was configured to anticoagulation. A solution of $0.2 \mathrm{~mL}$ anticoagulant was added to five centrifuge tubes, each containing $10 \mathrm{~mL}$ leaching solution ((a) PDLLA; (b) PDLLA/ $\beta$-TCP; (c) PDLLA $/ \beta-T C P /$ collagen; (d) autologous nerve groups; (positive groups) distilled water; (negative groups) $\mathrm{NaCl}$ solution), respectively. All centrifuge tubes were put in a thermostatic water bath at $37{ }^{\circ} \mathrm{C}$ for $1 \mathrm{~h}$ with the positive groups (distilled water) and negative groups ( $\mathrm{NaCl}$ solution); then the supernatants of the centrifuge tubes were sucked up and tested by the automatic enzyme instrument (Thermo Labsystems 1500-206, USA) at $542 \mathrm{~mm}$ after centrifugation (3000 rpm, $5 \mathrm{~min}$ ), and the optical absorbance was recorded.

$$
\text { Hemolysis ratio/\% }=(A-B) /(C-B) \times 100 \%
$$

( $A, B$ and $C$ represent the absorbance value of text groups, negative groups and positive groups).

\section{Experimental animal and surgical procedure}

The Disease Control and Prevention of Hubei Province (China) (license no. SCXK (E) 2015-0018) supplied forty 8 week-old, female Wistar (200-250 g) rats, which were randomly distributed into four groups ((a) PDLLA; (b) PDLLA/ $\beta$-TCP; (c) PDLLA/ $\beta$ TCP/collagen; (d) autologous nerve groups), each group having 10 rats. The composite films (PDLLA, PDLLA $/ \beta$-TCP, PDLLA $/ \beta$ TCP/collagen) were stuck into conduits (14 mm length; $2 \mathrm{~mm}$ inner diameter; $0.2 \mathrm{~mm}$ thickness) using dichloromethane. The Wistar rats were anesthetized with $10 \mathrm{~mL} \mathrm{~kg}^{-1}$ pentobarbital sodium through intraperitoneal injection method, after which the hair on their right legs were shaved clean, daubed with a tincture of iodine and made skin incision following the skin incision, then cut off $5 \mathrm{~mm}$ sciatic nerve until the broken end natural retraction $10 \mathrm{~mm}$. Then, both the proximal and distal stumps were sutured with 9-0 absorbable sutures to a depth of $2 \mathrm{~mm}$ into the conduits. In autologous nerve groups where the defect nerves were bridged with autologous nerves and connected by 9-0 absorbable sutures (PGA, Shanghai), the surgical incisions were sutured with surgery sutures. All rats were put 
into an animal house after disinfection, and the temperature was maintained at $23{ }^{\circ} \mathrm{C}$. In this article, autograft groups were set as control groups, that is widely used in clinics as the gold standard. ${ }^{56}$

\section{Gross view}

The rats were observed at regular intervals after the surgical operation for wound healing, the degree of swelling, gait, etc. Attention was paid to observe whether the presence of glioma and tissue adhesion during the process that regenerated nerves were removed from rats.

\section{Hematoxylin-eosin staining}

The conduits were opened after 3 months' implantation; subsequently, regenerated nerves, normal nerves and soleus muscles around the sciatic nerves were collected after cleaning the samples with normal saline. The regenerated nerves and soleus muscles were put into immobilizing solution containing $1 \%$ glutaraldehyde, $0.1 \mathrm{M}$ cacodylate and $1 \%$ paraformaldehyde, dehydrated in a rank, and embedded with paraffin following standard histological techniques. The samples of the nerves and soleus muscles were cut into slices $(0.5-1 \mu \mathrm{m})$ and stained with hematoxylin and eosin, and the slices of hematoxylin and eosin were observed and analyzed using an electron microscope (TE2000U, NiKon).

\section{Immunofluorescence staining}

The antibody of DAPI (labeling Schwann cells) and mouse antineurofilament 200 (labeling regenerated neurofilament) were used in immunofluorescence. The antibody of DAPI and mouse anti-neurofilament 200 were dissolved in the mixed liquor (PBS and goat serum) prepared as dye liquor. Sections of the samples were added to a solution of goat serum at $37^{\circ} \mathrm{C}$ for $30 \mathrm{~min}$. Then these sections were taken out from the goat serum and added to the dye liquor for overnight incubation at $4{ }^{\circ} \mathrm{C}$. The process of staining was carried out under the exits of humidor. The nerve tissue sections were carefully washed with PBS after incubation, then put in dye liquor for incubation at $4{ }^{\circ} \mathrm{C}$. When the process of immunofluorescence was completed, the slices of DAPI and mouse anti-neurofilament 200 were observed and analyzed using the electron microscope (TE2000U, NiKon).

\section{Statistical analysis}

The experimental data was expressed as mean \pm standard error. Statistical significance analysis was carried out using SPSS10.0 (SPSS Inc, Chicago, IL, USA). A value of $P \leq 0.05$ shows statistical significance.

\section{Results and discussion}

\section{Fourier transforms infrared spectroscopy (FTIR)}

Fig. 1 illustrates the infrared absorption spectra of the polymer films. The absorption band at $1755 \mathrm{~cm}^{-1}$ is the characteristic peak of the ester $(\mathrm{C}=\mathrm{O})$. The bands at $2995 \mathrm{~cm}^{-1}$ and $1455 \mathrm{~cm}^{-1}$ could be assigned to the stretching of the $\mathrm{C}-\mathrm{H}$ bond of methyl $\left(\mathrm{CH}_{3}\right)$.
The band located at $2945 \mathrm{~cm}^{-1}$ represents the characteristic peak of $\mathrm{CH}_{2}$, the band located at $1090 \mathrm{~cm}^{-1}$ is the characteristic peak of $\mathrm{COO}^{-}$. The above absorption bands reveal the existence of PDLLA in samples a, b and c. The bands at 606 and $552 \mathrm{~cm}^{-1}$ could be ascribed to the $\mathrm{PO}_{4}{ }^{3-}$ from $\beta$-TCP and they appeared in sample $\mathrm{b}$ and $\mathrm{c}$, revealing the embedding $\beta$-TCP in the PDLLA matrix. The typical amide I (ca. $\left.1650 \mathrm{~cm}^{-1}\right)$, amide II $\left(c a .1544 \mathrm{~cm}^{-1}\right)$ and amide III ( $\left.c a .1336 \mathrm{~cm}^{-1}\right)$ absorption bands could be observed in both sample $\mathrm{c}$ and pure collagen, indicating the successful incorporation of collagen to the PDLLA/ $\beta$-TCP matrix.

\section{Scanning electron microscopy (SEM)}

Fig. 2 shows the morphologies of the polymer films (sample a1, b1 and c1). Uniform fibers are observed for all three samples as revealed in figures a1, b1 and c1. Figures a2, b2 and c2 illustrate the SEM images of sample a1, b1 and c1 with a higher magnification. As measured from these images, the diameters of fibers in sample a1, b1 and c1 are $0.91 \pm 0.12 \mu \mathrm{m}, 0.87 \pm 0.13$ $\mu \mathrm{m}$ and $0.99 \pm 0.18 \mu \mathrm{m}$, respectively. It is also possible to measure the pore size from images shown in figures a2, b2 and $\mathrm{c} 2$, and the results for samples $\mathrm{a} 1, \mathrm{~b} 1$ and $\mathrm{c} 1$ are $2.51 \pm 0.53 \mu \mathrm{m}$, $2.72 \pm 0.61 \mu \mathrm{m}$ and $3.01 \pm 0.74 \mu \mathrm{m}$, respectively. The mean diameters of the pores in these films are less than $10 \mu \mathrm{m}$ and greater than $1 \mu \mathrm{m}$. These films have bionic structures that can not only permit the transportation of nerve growth factor and glucose but also block the entry of lymphatic and fibroblasts. As a result, the growth of glioma could be effectively prevented in the semi-permeable structure. ${ }^{57}$ The structures of these electrospinning films are believed to provide a suitable microstructure for the recovery of the peripheral nerves. Due to the instability and swelling of collagen, the fiber diameter and the filmpore size of $c$ were larger than those of $a$ and $b$ during the process of electrospinning.

\section{Mechanical property}

Fig. 3 illustrates the mechanical performance of the obtained polymer films. Fig. 3A shows the tensile strength of sample a, $\mathrm{b}$ and c. Fig. 3B shows the elongation at break of these three samples. Clearly, the incorporation of both $\beta$-TCP and collagen

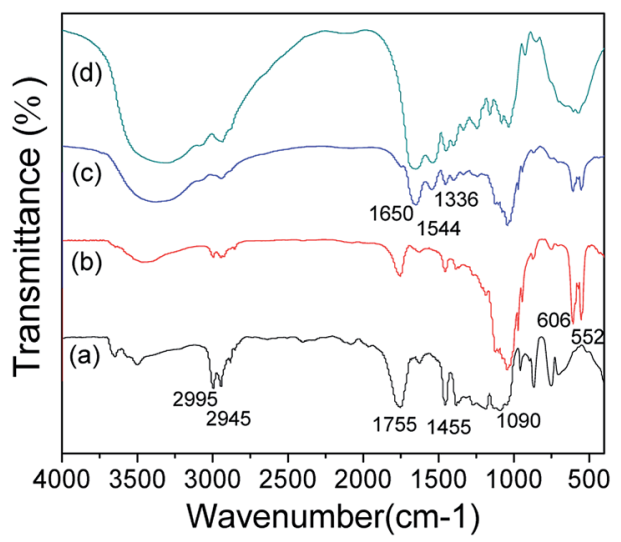

Fig. 1 FTIR spectroscopy of different groups. (a) PDLLA; (b) PDLLA/ $\beta$ TCP; (c) PDLLA/ $\beta$-TCP/collagen; (d) collagen. 

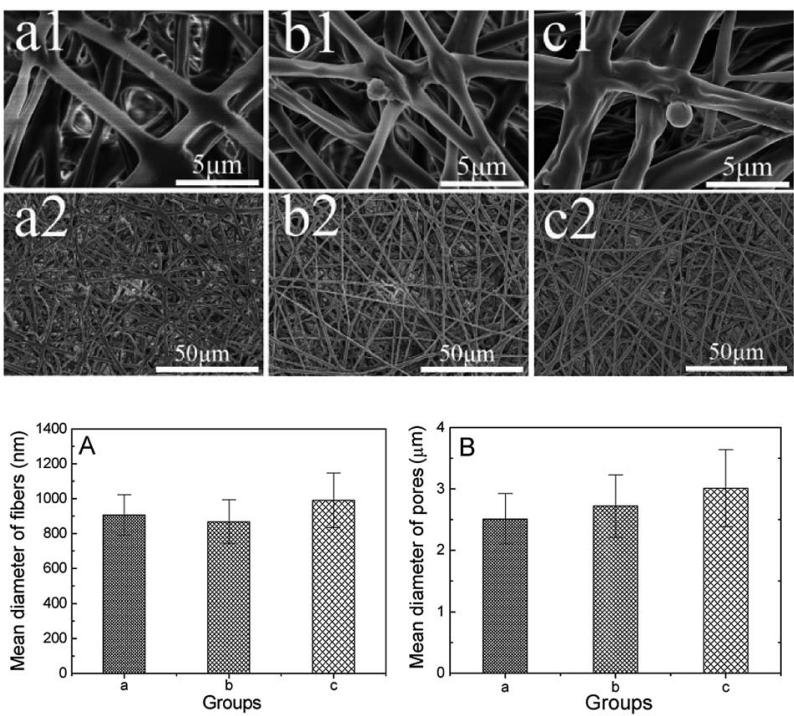

Fig. 2 Images of electrospinning films and the mean diameter of fibers and pores. (a1, a2) PDLLA; (b1, b2) PDLLA/ $\beta$-TCP; (c1, c2) PDLLA/ $\beta$ $\mathrm{TCP} /$ collagen; $(\mathrm{A})$ mean diameter of fibers; $(\mathrm{B})$ mean diameter of pores.

reduced the tensile strength and elongation percentage, leading to poor mechanical performance. Previous studies have shown that the polymer films need a tensile strength of at least $1.3 \mathrm{MPa}$ for their applications in tissue engineering..$^{58}$ In this study, all three samples met these criteria thanks to the novel mechanical property of the PDLLA matrix. $\beta$-TCP is a type of small solid particle material which can't form a continuous fiber via electrospinning, so $\beta$-TCP is attached to the surface of the electrospinning fibers or embedded in electrospinning fibers. There is no corresponding chemical bond, and mechanical effects between $\beta$-TCP and the electrospinning fibers can't play an effective role in enhancing the mechanical strength of PDLLA $/ \beta$ $\mathrm{TCP} /$ collagen. Collagen is the main component of the extracellular matrix that can be recognized by the cells, but the mechanical properties of collagen are poor; hence, the addition of collagen will reduce the overall mechanical properties of PDLLA $/ \beta$-TCP/collagen. In this study, the molecular weight of PDLLA was low, its internal molecular chain was short and the force between molecules was weak; thus, the addition of collagen and $\beta$-TCP led to the formation of electrospinning fibers that were discontinuous and unstable, which further
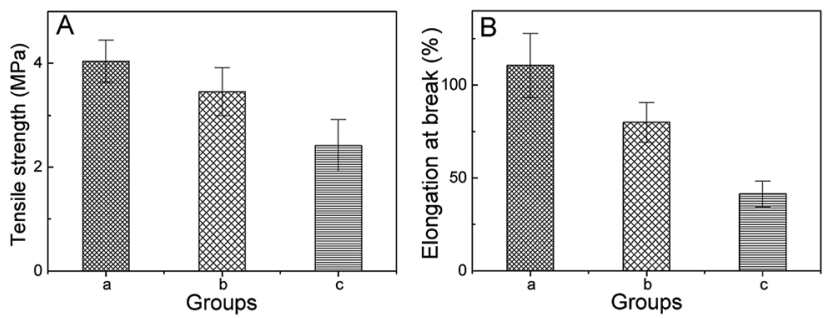

Fig. 3 Mechanical property with different groups. (A) Tensile strength; (B) elongation at break; (a) PDLLA; (b) PDLLA/ $\beta$-TCP; (c) PDLLA/ $\beta$-TCP/ collagen. reduced the overall mechanical properties of PDLLA $/ \beta-T C P /$ collagen.

\section{Biodegradation}

Fig. 4 shows degradation of the polymer films. As shown in Fig. $4 \mathrm{~A}$, the mass loss rate of different samples is in the order of $4 \mathrm{c}>4 \mathrm{~b}>4 \mathrm{a}$, indicating that the addition of both $\beta$-TCP and collagen accelerated the degradation process. For all these samples, degradation was faster in the first 56 days and then slower later on. Such degradation was beneficial for the recovery of injured nerves. ${ }^{59}$ Fig. $4 \mathrm{~B}$ illustrates the $\mathrm{pH}$ values of the environment during the degradation of different samples. The degradation of these polymer films is accompanied by a drop in $\mathrm{pH}$ values. For sample $4 \mathrm{~b}$ and $4 \mathrm{c}$, a lower drop was observed after the incorporation of $\beta$-TCP, whose dissolution can neutralize the acidic environment. ${ }^{23}$ In the first three weeks, the $\mathrm{pH}$ values of sample $4 \mathrm{~b}$ and $4 \mathrm{c}$ were close but the $\mathrm{pH}$ value of sample $4 \mathrm{c}$ was significantly higher than that of sample $4 \mathrm{~b}$ since the fourth week. Such phenomena could be related to the higher mass loss rate of sample $4 \mathrm{c}$. The $\mathrm{pH}$ values of the environments of all these polymer films are suitable for the growth of cells in terms of nerve recovery.

PDLLA is a type of polyester and its degradation is mainly through ester group hydrolysis, which is divided into two stages. First, the polymer backbone begins to break and the molecular weight is greatly reduced due to the penetration of water molecules. Second, the ester groups on the molecular chain are exposed to water and hydrolysis takes place. The process of degradation in $\mathrm{a}, \mathrm{b}$ and $\mathrm{c}$ occurs rapidly at first and slow down later. The degradation of $\beta$-TCP is mainly divided into two parts. First, degradation with the body fluids affects the implanted tissue that contains some acidic metabolites that promote the dissolution of $\beta$-TCP, which maintains the balance of $\mathrm{pH}$ in the conduit microenvironment. Second, cell phagocytosis and absorption affect a large number of fibroblasts, macrophages and other cells that are involved in the absorption of $\beta$-TCP; hence, the mass loss rate of $b$ and $c$ are faster than that of $a$. Collagen is one of the commonly used natural polymers, which has superior degradation performance and cytocompatibility in the body. As the degradation rate of collagen is higher than that of PDLLA and $\beta$-TCP, the mass loss rate of $\mathrm{c}$ is faster than those of $\mathrm{a}$ and $\mathrm{b}$. The $\mathrm{pH}$ of $\mathrm{c}$ is higher than that of $\mathrm{b}$ due to the neutralization of $\beta$-TCP and physiological regulation of cells.
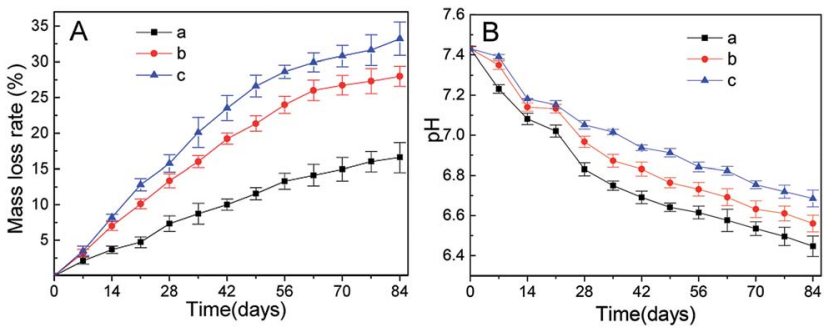

Fig. 4 Biodegradation of different groups. (A) Mass loss of different materials; (B) $\mathrm{pH}$ of different degradation liquids; (a) PDLLA; (b) PDLLA/ $\beta$-TCP; (c) PDLLA/ $\beta$-TCP/collagen. 
The degradation characteristics of each group are different when implanted in the body due to the different degradation mechanisms of each group.

\section{MTT}

As shown in Fig. 5 that the value of absorbance in $5 \mathrm{c}$ and negative groups are higher than those in $5 \mathrm{a}$ and $5 \mathrm{~b}$, for the relation between absorbance values and cytoactive are positive correlation, so the cytoactive is very active that the RSC96 cells adhesion, viability and proliferation in $5 \mathrm{c}$ are more superior than $5 \mathrm{a}$ and $5 \mathrm{~b}$ that is beneficial to repair injured nerves. The absorbance value between $5 \mathrm{~b}$ and $5 \mathrm{c}$ showed a significant difference $(* p<0.05)$ between days 3 and 5 , while the absorbance value between $5 \mathrm{c}$ and negative groups (blank control) showed no significant differences $\left({ }^{*} p>0.05\right)$ in the same interval, which indicated the low toxicity of $5 \mathrm{c}$ which was close to that of negative groups. According to the cell toxicity grade (Table 1), the relative growth rate of RSC96 cells in 5c is the highest among three conduit groups and is close that of to negative groups; hence, the cell toxicity grade of $5 \mathrm{c}$ (relative growth rate $=93.5 \%$ ) was ranked at grade 1 , which indicates the cell toxicity of $5 \mathrm{c}$ is so low that it meets the requirement of biomedical materials. The leaching solution of contains $\beta$-TCP and collagen small molecules, which is conducive to RSC96 cell growth and promotes RSC96 cell proliferation at a certain concentration; hence, the cell rate of $\mathrm{c}$ is higher than those of a and $b$ (Fig. 5).

\section{Hemolytic test}

The blood compatibility of materials is a significant index to judge whether such materials can be used as biomedical materials. The hemolysis ratio of national standard (GB-T16886 15-2003) used in medical implant materials should be lower than $5 \%$; hence, the hemolysis test is necessary. The color of positive groups is red $(+)$ because the addition of distilled water caused erythrocyte rupture and changed the shape of the erythrocyte. The sample groups $(6 a ; 6 b ;$ and $6 c)$ and the negative groups were colorless and the erythrocyte deposited in the bottom of the tubes were not broken, which indicated a low rate

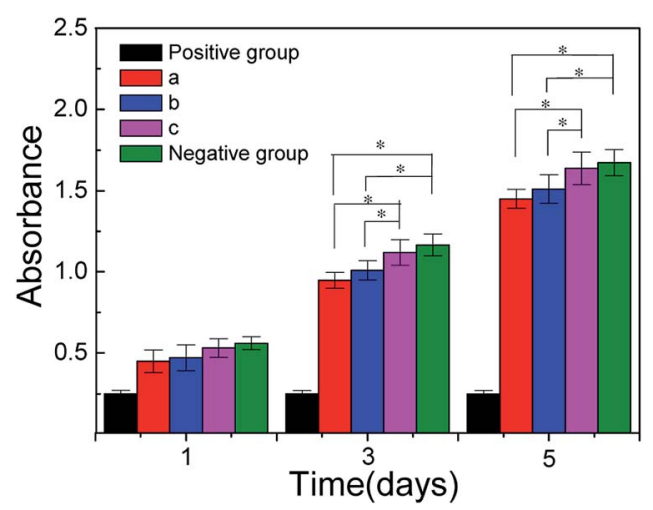

Fig. 5 Results of MTT tests with different groups. (a) PDLLA; (b) PDLLA/ $\beta$-TCP; (c) PDLLA/ $\beta$-TCP/collagen; (positive) DMSO; (negative) blank control.
Table 1 The standard of cytotoxicity classification ${ }^{a}$

\begin{tabular}{ll}
\hline Relative growth rate $(\%)$ & Cell toxicity grade \\
\hline$\geq 100$ & 0 \\
$75-99$ & 1 \\
$50-74$ & 2 \\
$25-49$ & 3 \\
$1-24$ & 4 \\
$\leq 0$ & 5
\end{tabular}

${ }^{a}$ Relative growth rate/\% $=(A-B) /(C-B) \times 100 \%(A, B$ and $C$ represent the absorbance value of test groups, positive groups and negative groups).

of erythrocyte rupture (Fig. 6). The hemolysis ratio of $6 \mathrm{c}$ was the least among the three by calculation from the absorbance value, and the hemolysis rate in $6 \mathrm{c}$ met the national standards of medical implant material. Nonhemolysis materials are defined as having $0-2 \%$ hemolysis rate, according to the American Society for Testing and Materials, ${ }^{60}$ so the three groups $(6 \mathrm{a}, 6 \mathrm{~b}$, $6 \mathrm{c}$ ) are nonhemolysis materials. $6 \mathrm{c}$ had the lowest hemolysis rate compared to $6 \mathrm{a}$ and $6 \mathrm{~b}$ indicating that it has the best biocompatibility compared to $6 \mathrm{a}$ and $6 \mathrm{~b}$ when used in vivo. The performance of the material has a significant influence on cell affinity when the material surface makes contact with nerve cells. The cell membrane can specifically recognize the protein on the material as proteins have several amino acids. Collagen also contains several amino acids, which can make contact red cells efficiently and not change the osmotic pressure of the solution. $\beta$-TCP could improve the material hydrophilicity that is conducive to erythrocyte survival, so $\mathrm{c}$ had a lower hemolysis rate than a and $\mathrm{b}$. These results imply that $6 \mathrm{c}$ with the lowest hemolysis rate can be used in vivo securely (Table 2).

\section{Gross view}

The death of rats did not occur during the period of recovery. All rats had difficulty in walking and had a slight limp and showed poor mental state within 1 week of observations. None of wounds were infected or bitten by rats, part of the toes showed inflammation and ulceration to some degree in 2 weeks, and all rats exhibited myophagism after 1 month. The PDLLA/ $\beta$-TCP/ collagen and autologous groups showed an evident feeling of

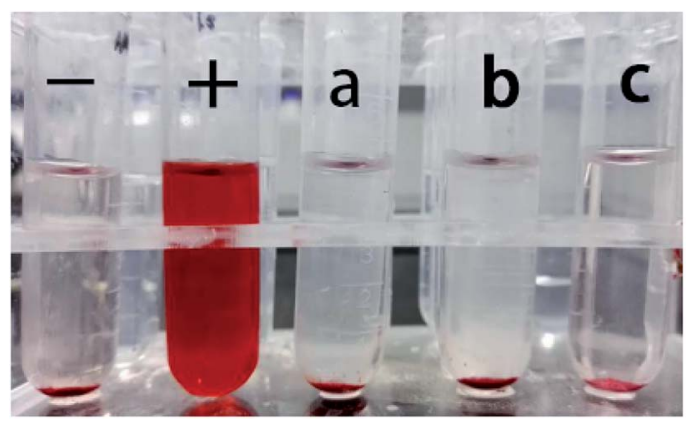

Fig. 6 Images of the hemolytic test; $(-)$ negative groups; $(+)$ positive groups; (a) PDLLA; (b) PDLLA/ $\beta$-TCP; (c) PDLLA/ $\beta$-TCP/collagen. 
Table 2 The rate of hemolysis ratio with different materials

\begin{tabular}{llc}
\hline Groups & Samples & Hemolysis ratio/\% \\
\hline a & PDLLA & $0.46 \pm 0.19$ \\
$\mathrm{~b}$ & PDLLA/ $/$-TCP & $0.27 \pm 0.12$ \\
$\mathrm{c}$ & PDLLA $/$-TCP/collagen & $0.20 \pm 0.09$ \\
- & Negative groups & $0 \pm 0.03$ \\
+ & Positive groups & $1 \pm 0.02$
\end{tabular}

pain in the right leg compared to the PDLLA and PDLLA/ $\beta$-TCP groups, and the ulceration of toes had disappeared after 2 months. PDLLA/ $\beta$-TCP/collagen and the autologous groups had no difficulty in walking, and the walking ability of the rats in the PDLLA $/ \beta$-TCP/collagen group was better than that of rats in the PDLLA and PDLLA/ $\beta$-TCP groups after surgical operation for 3 months. The recovery of muscle in the PDLLA $/ \beta$-TCP/collagen and autologous groups was significantly improved compared to that in the PDLLA and PDLLA/ $\beta$-TCP groups.

The remaining conduits were removed and the regenerated nerves were taken out 3 months after implantation. No conglutination was observed between conduits and the surrounding tissues, the neuroma did not appear in the regenerated nerves, and the ends of the distal and proximal nerves were connected completely through regenerated nerves. The regenerated nerves indicated that the nerve continuity was fully recovered in all groups from the macroscopic observation. The thickness of regenerated nerves in the PDLLA $/ \beta$-TCP/ collagen group was higher than that in the PDLLA and PDLLA/ $\beta$-TCP groups. The shape of regenerated nerves was more regular and well distributed in the PDLLA/ $\beta$-TCP/collagen group than in the groups PDLLA and PDLLA/ $\beta$-TCP groups.
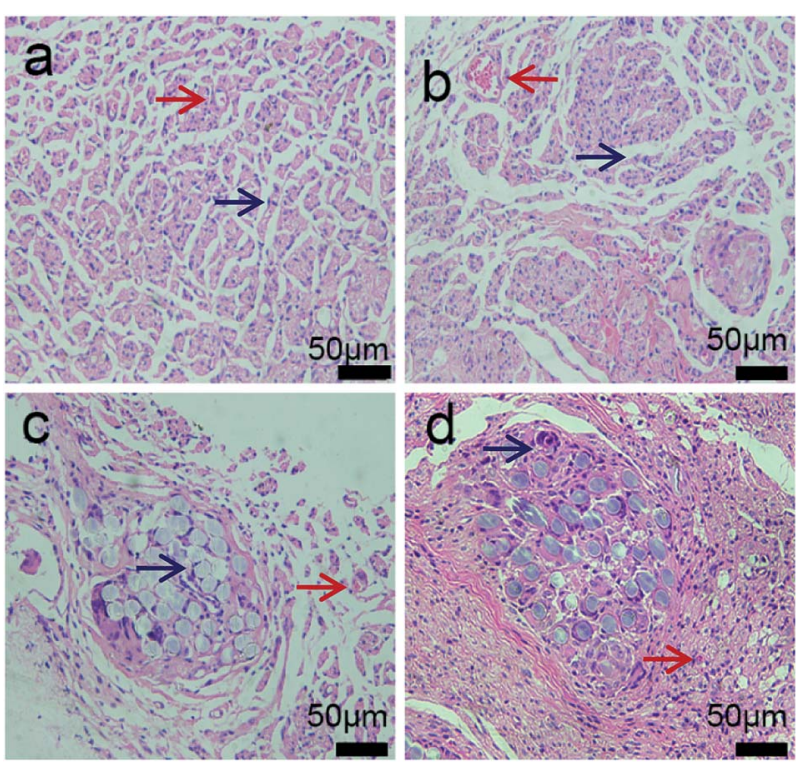

Fig. 7 Morphology of regenerated sciatic nerves in rats at 3 months after surgical operation (hematoxylin-eosin staining). (a) PDLLA; (b) PDLLA/ $\beta$-TCP; (c) PDLLA/ $\beta$-TCP/collagen; (d) autologous groups; (red arrows) blood vessels; (blue arrows) the nucleus of Schwann cells.

\section{Hematoxylin-eosin (HE) staining}

To better evaluate the recovery of injured nerves, the middle segment of regenerated nerves was dyed with hematoxylineosin staining 3 months after the surgical operation. All rats were in good health and no deaths were reported; wound infection and severe tissue reactions happened during the recovery. Arrows indicated in red and blue colors correspond to blood vessels and the nucleus of Schwann cells, respectively. Blood vessels existed in the composite conduit groups $(7 \mathrm{a} ; \mathrm{7b}$; $7 \mathrm{c} ; 7 \mathrm{~d})$, which indicated that the blood vessels generate and provide nutrients to injured nerves during the process of recovery. Schwann cells existed in the four groups and promoted the growth of injured nerves. The myelinated nerve fibers in $7 \mathrm{a}$ and $7 \mathrm{~b}$ were distributed in arrangement with thin myelin sheath in a way and most irregular connective tissues in growth. The nerve fibers of regenerated nerves in $7 \mathrm{c}$ are more circular in shape, better-proportioned in size and arranged more densely than in $7 \mathrm{a}$ and $7 \mathrm{~b}$. There are a large number of fascicular structures scattered throughout nerves in $7 \mathrm{c}$. Based on the morphology observation, the myelinated nerve fibers in $7 \mathrm{c}$ are greater in number and more intensive than those in $7 \mathrm{a}$ and $7 \mathrm{~b} 3$ months after implantation. Nerve fibers were in greater proportion in $7 \mathrm{c}$ than in $7 \mathrm{a}$ and $7 \mathrm{~b}$, and this proportion was close to what was present in $7 \mathrm{~d}$ (Fig. 7).
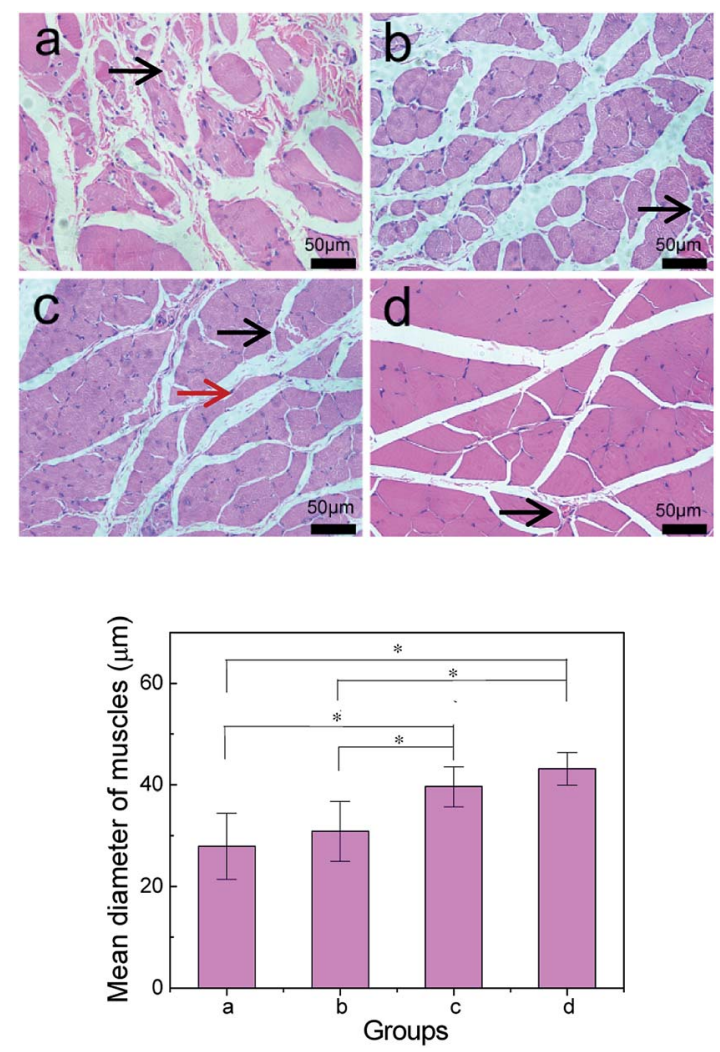

Fig. 8 Morphology and mean diameter of soleus muscles in rats at 3 months after sciatic nerve injury (hematoxylin-eosin staining). (a) PDLLA; (b) PDLLA/ $\beta$-TCP; (c) PDLLA/ $\beta$-TCP/collagen; (d) autologous groups; (black arrow) myocytes atrophy; (red arrow) connective tissues; ${ }^{*} P<0.05$ 
Fig. 8 shows the form of soleus muscles, which around injured sciatic nerves shows the condition of nerve recovery that plays a significant role in the analyzed sciatic nerve function in rats. Soleus muscles around the injured sciatic nerves were collected and dyed with hematoxylin-eosin 3 months after implantation. Muscular atrophy was observed in the four groups wherein the myocytes (black arrow) were degenerated, shrunken in different degrees and loosely packed. The soleus muscles shown in $8 \mathrm{a}$ and $8 \mathrm{~b}$ had obvious atrophy from the form compared to those shown in $8 \mathrm{c}$ and $8 \mathrm{~d}$ after surgical operation, and the mean diameter of muscle fibers were smaller in $8 \mathrm{a}$ and $8 \mathrm{~b}$. The morphology of myocytes shown in $8 \mathrm{c}$ was better than those of myocytes shown in $8 \mathrm{a}$ and $8 \mathrm{~b}$, but worse than that shown in $8 \mathrm{~d}$. We found an obvious existence of regenerated soleus muscles and connective tissues in $8 \mathrm{c}$ compared to that in $8 \mathrm{a}$ and $8 \mathrm{~b}$ due to the addition of $\beta$-TCP and collagen. $\beta$-TCP can neutralize acid, which is produced by PDLLA and collagen, and this maintains the neutral microenvironment between the soleus muscles and nerve conduits. Collagen can improve the cellular affinity of nerve conduits, and this is beneficial to the recovery of muscle atrophy. The mean diameter of muscle fibers in $8 \mathrm{c}(39.62 \pm 3.91 \mu \mathrm{m})$ was 1.28 times larger than those of muscle fibers in $8 \mathrm{~b}(30.89 \pm 5.89 \mu \mathrm{m})(P<0.05)$ and was smaller than those of muscle fibers in $8 \mathrm{~d}(41.13 \pm 3.22 \mu \mathrm{m})$ $(P>0.05)$; therefore, $8 \mathrm{c}$ had the largest mean diameter of muscle fibers among the three conduit groups $(8 \mathrm{a} ; 8 \mathrm{~b} ; 8 \mathrm{c})$. Mean diameter of muscle fibers in $8 \mathrm{c}$ was close to the value in $8 \mathrm{~d}$. These results indicate that the recovery of sciatic nerve function in $8 \mathrm{c}$ is closer to that observed in the autologous groups.
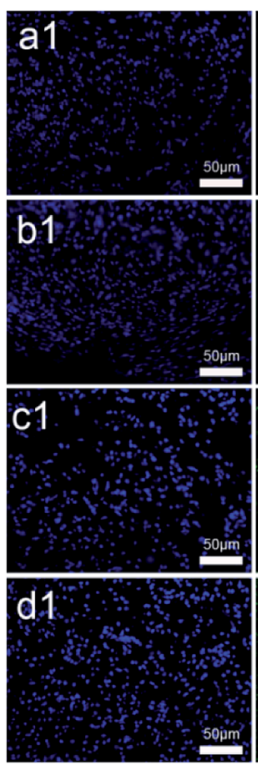
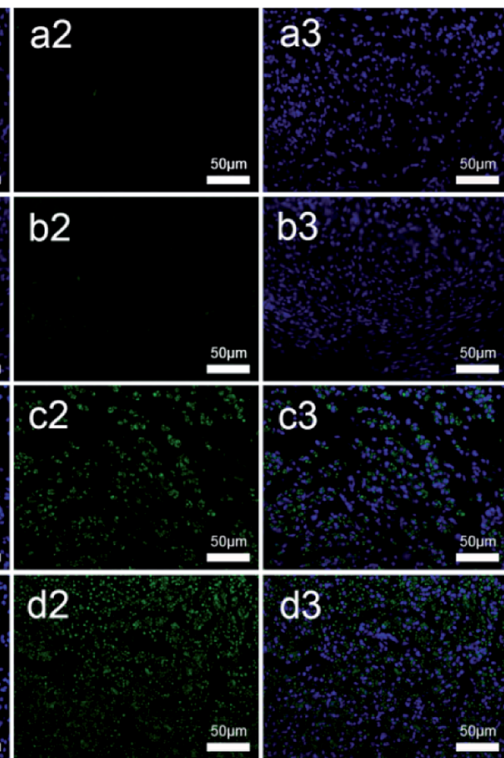

\section{Immunofluorescence staining}

Immunofluorescence staining is an effective way to observe myelin growth and maturity of nerves. Fig. 9 shows blue and green color in all four groups (9a; 9b; 9c; 9d), which indicates that the regenerated sciatic nerves showed growth of Schwann cells and neurofilaments during the recovery of injured nerves. Confocal laser scanning microscopy revealed that the Schwann cells (blue) were plump and the neurofilaments (green) were dot-like in appearance in all groups at 3 months after surgery. The density of Schwann cells in all groups (a1; b1; c1; d1) was high, while the density of regenerated neurofilaments in c2 was more than that in $\mathrm{a} 2$ and b2, but less than that in $\mathrm{d} 2$. The morphology of regenerated neurofilaments in c2 was more circular and packed in arrangement than those in a2 and b2, while it was closer in value to that in $\mathrm{d} 2$. Schwann cells grow around the regenerated neurofilaments, which enhance nerve regeneration through secreted nerve growth factor, brain derived neurotrophic factor, etc.

\section{Conclusion}

We have successfully prepared PDLLA/ $\beta$-TCP/collagen conduits via the electrospinning method and investigated its biocompatibility and peripheral nerve regeneration. PDLLA, $\beta$-TCP and collagen exist in PDLLA/ $\beta$-TCP/collagen conduit through the relevant absorption peaks shown from FTIR. The SEM image shows that the film of PDLLA/ $\beta$-TCP/collagen has a bionic structure that can not only permit the transportation of nerve growth factor and glucose but also block the entry of lymphatic and fibroblasts. The mass loss rate and the $\mathrm{pH}$ of PDLLA $/ \beta-\mathrm{TCP} /$ collagen are suitable for nerve regeneration. MTT and hemolytic tests showed that PDLLA/ $\beta$-TCP/collagen has better biocompatibility and efficiency compared to PDLLA and PDLLA/ $\beta$-TCP. PDLLA/ $\beta$-TCP/collagen has well biocompatibility, which meet the standard as a biomedical material implant in animals. The PDLLA/ $\beta$-TCP/collagen nerve conduit could repair $10 \mathrm{~mm}$ sciatic nerve defects in rats at 3 months from gross view, which is close to autologous groups. We observed that the morphology of regenerated nerves in the PDLLA/ $\beta$-TCP/collagen groups was close to that of regenerated nerves in the autologous groups and better than that of regenerated nerves in the PDLLA and PDLLA/ $\beta$-TCP groups. Similarly, the mean diameter of muscle fibers in the PDLLA $/ \beta$-TCP/collagen groups was larger than that of muscle fibers in the PDLLA/ $\beta$-TCP $(P<0.05)$ group and close to that muscle fibers in the autologous groups $(P>0.05)$ based on a histological observation. To serve as a suitable biomaterial for peripheral nerve regeneration with the least delay possible, further investigations should be done on PDLLA/ $\beta$-TCP/collagen.

\section{Conflicts of interest}

There are no conflicts to declare.

\section{Acknowledgements}

sciatic nerve injury (immunofluorescence staining). (a1, a2, a3) PDLLA (b1, b2, b3) PDLLA/ $\beta$-TCP; (c1, c2, c3) PDLLA/ $\beta$-TCP/collagen; (d1, d2, d3) autologous groups; (blue) the nuclei of Schwann cells; (green) regenerated neurofilaments.
This work was supported by the Hong Kong, Macao and Taiwan Science \& Technology Cooperation Program of China (No. 
2015DFH30180), the Key Technology Research Plan of Wuhan Municipality (No. 2014060202010120) and the Science and Technology Support Program of Hubei Province (No. 2015BCE022).

\section{Notes and references}

1 Y. Z. Bian, Y. Wang, G. Aibaidoula, G. Q. Chen and Q. Wu, Biomaterials, 2009, 30, 217-225.

2 Q. Li, L. Ma and C. Gao, J. Mater. Chem. B, 2015, 3, 89218938.

3 C. Gumera, B. Rauck and Y. Wang, J. Mater. Chem., 2011, 21, 7033-7051.

4 S. Kehoe, X. F. Zhang and D. Boyd, Injury, 2012, 43, 553572.

5 J. Cao, Z. Xiao, W. Jin, B. Chen, D. Meng, W. Ding, S. Han, X. Hou, T. Zhu and B. Yuan, Biomaterials, 2013, 34, 13021310.

6 N. Hu, H. Wu, C. Xue, Y. Gong, J. Wu, Z. Xiao, Y. Yang, F. Ding and X. Gu, Biomaterials, 2012, 34, 100-111.

7 C. Miller, H. Shanks, A. Witt, G. Rutkowski and S. Mallapragada, Biomaterials, 2001, 22, 1263-1269.

8 G. R. Evans, K. Brandt, S. Katz, P. Chauvin, L. Otto, M. Bogle, B. Wang, R. K. Meszlenyi, L. Lu and A. G. Mikos, Biomaterials, 2002, 23, 841-848.

9 Y. S. Chen, J. Y. Chang, C. Y. Cheng, F. J. Tsai, C. H. Yao and B. S. Liu, Biomaterials, 2005, 26, 3911-3918.

10 M. R. Ahmed, S. Vairamuthu, M. Shafiuzama, S. H. Basha and R. Jayakumar, Brain Res., 2005, 1046, 55-67.

11 E. Schnell, K. Klinkhammer, S. Balzer, G. Brook, D. Klee, P. Dalton and J. Mey, Biomaterials, 2007, 28, 3012-3025.

12 G. Lundborg, L. B. Dahlin, N. Danielsen, R. H. Gelberman, F. M. Longo, H. C. Powell and S. Varon, Exp. Neurol., 1982, 76, 361-375.

13 N. L. Mligiliche, Y. Tabata and C. Ide, East Afr. Med. J., 1999, 76, 400-406.

14 T. Kauppila, E. Jyväsjärvi, T. Huopaniemi, E. Hujanen and P. Liesi, Exp. Neurol., 1993, 123, 181-191.

15 J. Zeng, X. Xu, X. Chen, Q. Liang, X. Bian, L. Yang and X. Jing, J. Controlled Release, 2003, 92, 227-231.

16 D. K. Gilding and A. M. Reed, Polymer, 1979, 20, 1459-1464.

17 D. Li, X. Pan, B. Sun, T. Wu, W. Chen, C. Huang, Q. Ke, H. A. EI-Hamshary, S. Al-Deyab and X. Mo, J. Mater. Chem. $B, 2015,3,8823-8831$.

18 T. Wu, D. Li, Y. Wang, B. Sun, D. Li, Y. Morsi, H. ElHamshary, S. S. Al-Deyab and X. Mo, J. Mater. Chem. B, 2017, 5, 3186-3194.

19 C. Gardin, L. Ferroni, L. Favero, E. Stellini, D. Stomaci, S. Sivolella, E. Bressan and B. Zavan, Int. J. Mol. Sci., 2012, 13, 6452-6453.

20 B. Sun, X. J. Jiang, S. Zhang, J. C. Zhang, Y. F. Li, Q. Z. You and Y. Z. Long, J. Mater. Chem. B, 2015, 3, 5389-5410.

21 H. Fu, Q. Fu, N. Zhou, W. Huang, M. N. Rahaman, D. Wang and X. Liu, Mater. Sci. Eng., C, 2009, 29, 2275-2281.

22 K. G. Carrasquillo, A. M. Stanley, J. C. Aponte-Carro, P. D. Jésus, H. R. Costantino, C. J. Bosques and G. Kai, J. Controlled Release, 2001, 76, 199-208.
23 J. R. Jones, S. Ahir and L. L. Hench, J. Sol-Gel Sci. Technol., 2004, 29, 179-188.

24 M. Azami, F. Moztarzadeh and M. Tahriri, J. Porous Mater., 2010, 17, 313-320.

25 M. P. Lutolf and J. A. Hubbell, Nat. Biotechnol., 2005, 23, 4755.

26 C. T. Laurencin, S. F. Elamin, S. E. Ibim, D. A. Willoughby, M. Attawia, H. R. Allcock and A. A. Ambrosio, J. Biomed. Mater. Res., 1996, 30, 133-138.

27 J. R. Jones and L. L. Hench, J. Biomed. Mater. Res., Part B, 2004, 68, 36-44.

28 W. Song, G. R. Mitchell and K. Burugapalli, RSC Adv., 2015, 1, 1-39.

29 B. Mammadov, M. Sever, M. Gecer, F. Zor, S. Ozturk, H. Akgun, U. H. Ulas, Z. Orhan, M. O. Guler and A. B. Tekinay, $R S C$ Adv., 2016, 6, 110535-110547.

30 E. Biazar, S. H. Keshel and M. Pouya, Neural Regener. Res., 2013, 8, 2501-2509.

31 M. Zhang, W. Lin, S. Li, X. Y. Shi, Y. Liu, Q. Guo, Z. Huang, L. Li and G. L. Wang, J. Nanosci. Nanotechnol., 2016, 16, 9413-9420.

32 B. Bondar, S. Fuchs, A. Motta, C. Migliaresi and C. J. Kirkpatrick, Biomaterials, 2008, 29, 561-572.

33 D. Y. Lee, B. H. Choi, J. H. Park, S. J. Zhu, B. Y. Kim, J. Y. Huh, S. H. Lee, J. H. Jung and S. H. Kim, J. Craniomaxillofac. Surg., 2006, 34, 50-56.

34 C. Zhou, Y. Hong and X. Zhang, Biomater. Sci., 2013, 1, 10121028.

35 B. B. Li, Y. X. Yin, Q. J. Yan, X. Y. Wang and S. P. Li, Neural Regener. Res., 2016, 11, 150-155.

36 W. Dai, J. Belt and W. M. Saltzman, Biotechnology, 1994, 12, 797-801.

37 J. F. Alvarez-Barreto, M. C. Shreve, P. L. Deangelis and V. I. Sikavitsas, Tissue Eng., Part A, 2007, 13, 1205-1217.

38 C. Deng, X. Chen, H. Yu, J. Sun, T. Lu and X. Jing, Polymer, 2007, 48, 139-149.

39 M. H. Ho, L. T. Hou, C. Y. Tu, H. J. Hsieh, J. Y. Lai, W. J. Chen and D. M. Wang, Macromol. Biosci., 2006, 6, 90-98.

40 A. E. Postlethwaite, J. M. Seyer and A. H. Kang, Proc. Natl. Acad. Sci. U. S. A., 1978, 75, 871-875.

41 F. J. O'Brien, B. A. Harley, I. V. Yannas and L. Gibson, Biomaterials, 2004, 25, 1077-1086.

42 C. P. Barnes, S. A. Sell, E. D. Boland, D. G. Simpson and G. L. Bowlin, Adv. Drug Delivery Rev., 2007, 59, 1413-1433.

43 H. C. Liang, Y. Chang, C. K. Hsu, M. H. Lee and H. W. Sung, Biomaterials, 2004, 25, 3541-3552.

44 D. A. Tonge, J. P. Golding, M. Edbladh, M. Kroon, P. E. Ekström and A. Edström, Exp. Neurol., 1997, 146, 81-90. 45 A. C. de Luca, J. S. Stevens, S. L. Schroeder, J. B. Guilbaud, A. Saiani, S. Downes and G. Terenghi, J. Biomed. Mater. Res., Part A, 2012, 101, 491-501.

46 Q. Yan, Y. Yin and B. Li, Biomed. Eng. Online, 2012, 11, 1-16. 47 V. Guarino, F. Causa and L. Ambrosio, Expert Rev. Med. Devices, 2007, 4, 405-418.

48 K. Rezwan, Q. J. Chen and A. R. Boccaccini, Biomaterials, 2006, 27, 3413-3431.

49 H. Boedtker and P. Doty, J. Phys. Chem., 1954, 58, 968-983. 
50 X. Liu, L. Smith, J. Hu and P. Ma, Biomaterials, 2009, 30, 2252-2258.

51 A. Subramanian, U. M. Krishnan and S. Sethuraman, J. Biomed. Sci., 2009, 16, 108.

52 A. L. Luis, J. M. Rodrigues, S. Amado, A. P. Veloso, P. A. Armadadasilva, S. Raimondo, F. Fregnan, A. J. Ferreira, M. A. Lopes and J. D. Santos, Microsurgery, 2007, 27, 125-137.

53 B. Das, P. Chattopadhyay, M. Mandal, B. Voit and N. Karak, Macromol. Biosci., 2013, 13, 126-139.

54 Y. Xia, Nanoscale, 2010, 2, 35-44.
55 T. Qiu, Y. Yin, B. Li, L. Xie, Q. Yan, H. Dai, X. Wang and S. Li, J. Biomed. Mater. Res., Part A, 2014, 102, 3734-3743.

56 S. Lee, K. Saetia, S. Saha, D. G. Kline and D. H. Kim, Neurosurg. Focus, 2011, 31, E10.

57 V. B. Doolabh, M. C. Hertl and S. E. Mackinnon, Rev. Neurosci., 1996, 7, 47-84.

58 C. You, Z. F. Zhang and X. Tong, China Plast. Ind., 2007, 35, 47-49.

59 D. H. Rice, F. D. Burstein and A. Newman, Arch. Otolaryngol. Head. Neck. Surg., 1985, 111, 259-261.

60 ASTM International, ASTM F 756-00 Standard Practice for Assessment of Hemolytic Properties of Materials, 2008. 